\title{
Generalized Classical and Quantum Dynamics Within a Nonextensive Approach
}

\author{
A. Lavagno \\ Dipartimento di Fisica, Politecnico di Torino, I-10129 Torino, Italy, \\ and Istituto Nazionale di Fisica Nucleare, Sezione di Torino, Italy
}

Received on 20 December, 2004

\begin{abstract}
On the basis of generalized classical kinetic equations, reproducing the stationary distribution of the Tsallis nonextensive thermostatistics, we formulate two generalized Schrödinger equations which satisfy the basic assumptions of the quantum mechanics under an appropriate generalization of the operator properties. Moreover, we study the generalization of the previously introduced dynamic equations in a relativistic regime and we apply our results to the study of the rapidity distribution in the relativistic heavy-ion collisions.
\end{abstract}

\section{Introduction}

Let us review some basic assumptions of the nonextensive thermostatistics that will be useful in view of the generalized classical and quantum dynamics we will describe.

Starting point of the Tsallis' generalization of the Boltzmann-Gibbs statistical mechanics is the introduction of a $q$-deformed entropy functional defined, in a phase space system, as [1]

$$
S_{q}=-k_{B} \int d \Omega p^{q} \ln _{q} p,
$$

where $k_{B}$ is the Boltzmann constant, $p=p(x, v)$ is the phase space probability distribution, $d \Omega$ stands for the corresponding phase space volume element and $\ln _{q} x=\left(x^{1-q}-1\right) /(1-$ $q$ ) is, for $x>0$, the $q$-deformed logarithmic function. For the real parameter $q \rightarrow 1$, Eq.(1) reduces to the standard Boltzmann-Gibbs entropy functional.

In the equilibrium canonical ensemble, under the constraints imposed by the probability normalization

$$
\int d \Omega p=1
$$

and the normalized $q$-mean expectation value of the energy [2]

$$
\langle E\rangle_{q}=\frac{\int d \Omega p^{q} H(x, v)}{\int d \Omega p^{q}},
$$

the maximum entropy principle gives the probability distribution $[2,3]$

$$
p(x, v)=\frac{f(x, v)}{Z_{q}},
$$

where

$$
f(x, v)=\left[1-(1-q) \beta\left(H(x, v)-\langle H\rangle_{q}\right)\right]^{1 /(1-q)}
$$

and

$$
Z_{q}=\int d \Omega f(x, v) .
$$

Let us note that, depending from the extremization procedure, in Ref.[2] the above factor $\beta$ is only proportional to the Lagrange multiplier, because of the probability distribution is self-referential, while, in Ref.[3], $\beta$ is actually the Lagrange multiplier associated to the energy constraint.

By using the definitions in Eqs. (4) and (6) into the relation $Z_{q}^{1-q}=\int d \Omega p^{q}$ [3], the following identity holds [4]

$$
\int d \Omega f(x, v) \equiv \int d \Omega f^{q}(x, v),
$$

and the normalized $q$-mean expectation value for a physical observable $A(x, v)$ can be expressed as

$$
\langle A\rangle_{q}=\frac{\int d \Omega f^{q} A(x, v)}{\int d \Omega f^{q}} \equiv \frac{1}{Z_{q}} \int d \Omega f^{q} A(x, v) .
$$

Therefore, the probability distribution and the $q$-mean value of an observable have the same normalization factor $Z_{q}$, as in the extensive statistical mechanics. Such a non-trivial property does not depend on the equilibrium frame but it comes from the normalization condition and holds at any time (if we require that the transport equation conserves the probability normalization or the number of particles). This observation will play a crucial role in the following discussion.

\section{From Fokker-Planck equation to nonextensive Schrödinger equation}

The linear Fokker-Planck equation in the velocity space can be written as

$$
\frac{\partial}{\partial t}[f(t, v)]=-\frac{\partial}{\partial v}\left[J(v)[f(t, v)]-D \frac{\partial}{\partial v}[f(t, v)]\right] .
$$


Of course, the stationary solution of the above equation depends from the explicit expression of the drift $J$ and the diffusion coefficient $D$. It is easy to see that if $J$ and $D$ depend on the deformation parameter $q$ by means the following relation

$$
\frac{\partial D_{q}}{\partial v}-\frac{\beta m v}{1-(1-q) \beta E} D_{q}+J_{q}=0,
$$

where $E=m v^{2} / 2$, the stationary solution is the power-law Tsallis distribution function $[1,5]$

$$
f_{q}(v)=[1-(1-q) \beta E]^{1 /(1-q)} .
$$

On the basis of the above observations and following the stochastic quantization procedure, the "nonextensive" linear Schrödinger equation can be written as

$$
i \hbar \frac{\partial \psi}{\partial t}=\hat{H}_{q} \psi
$$

where $\hat{H}_{q}$ is the $q$-deformed Hamiltonian

$$
\hat{H}_{q}=\frac{\hat{H}}{1-(1-q) \frac{i}{\hbar} \hat{H} t},
$$

$\hat{H}$ is the Hamiltonian operator and we have assumed that it contains no explicit time dependence. The right side of Eq.(12) must be seen as a power series of the operator $(1-q) i \hat{H} t / \hbar$ for $q \approx 1$. Equation (12) does not admit factorized solutions but can be integrated to find the wave function at any time $t_{b}$ from the state at any other time $t_{a}$ as follows

$$
\psi\left(t_{b}\right)=\hat{U}_{q}\left(t_{b}, t_{a}\right) \psi\left(t_{a}\right),
$$

where $t_{a}<t_{b}$ and we have introduced the generalized $q$ deformed time evolution operator

$$
\begin{aligned}
\hat{U}_{q}\left(t_{b}, t_{a}\right) & =\frac{\left[1-(1-q) \frac{i}{\hbar} \hat{H} t_{b}\right]^{1 /(1-q)}}{\left[1-(1-q) \frac{i}{\hbar} \hat{H} t_{a}\right]^{1 /(1-q)}} \\
& \equiv \frac{\exp _{q}\left(-\frac{i}{\hbar} \hat{H} t_{b}\right)}{\exp _{q}\left(-\frac{i}{\hbar} \hat{H} t_{a}\right)}
\end{aligned}
$$

In the second equivalence we have used the definition of the Tsallis $q$-deformed exponential

$$
e_{q}^{x}=[1+(1-q) x]^{1 /(1-q)},
$$

which satisfies the properties: $e_{q}\left(\ln _{q} x\right)=x$ and $e_{q}(x)$. $e_{q}(y)=e_{q}[x+y+(1-q) x y]$.

The $q$-deformed time evolution satisfies the fundamental composition law. In fact, if two time translations are performed successively, the corresponding operators $\hat{U}_{q}$ are related by

$$
\hat{U}_{q}\left(t_{b}, t_{a}\right)=\hat{U}_{q}\left(t_{b}, t_{c}\right) \hat{U}_{q}\left(t_{c}, t_{a}\right),
$$

for any $t_{c} \in\left(t_{a}, t_{b}\right)$. It is important to observe that the operator $\hat{U}_{q}$ is not a unitary operator in the common sense, however if we observe that the $q$-deformed exponential, defined in Eq.(16), satisfies the following properties

$$
\begin{aligned}
e_{q}^{x} e_{2-q}^{-x} & =1, \\
e_{q}^{i x}\left(e_{2-q}^{i x}\right)^{*} & =1 \Leftrightarrow\left(e_{2-q}^{i x}\right)^{*}=\left(e_{q}^{i x}\right)^{-1},
\end{aligned}
$$

it appears natural to define the $q$-adjoint of an operator $\hat{O}_{q}$ as

$$
\left\langle a\left|\hat{O}_{q}^{\dagger}\right| b\right\rangle=\left\langle b\left|\hat{O}_{2-q}\right| a\right\rangle^{*}
$$

On the basis of the above prescription, the $q$-deformed time evolution operator can be view as a q-unitary operator

$$
\hat{U}_{q}^{\dagger}\left(t_{b}, t_{a}\right)=\left[\hat{U}_{q}\left(t_{b}, t_{a}\right)\right]^{-1}
$$

and

$$
\left[\hat{U}_{q}\left(t_{b}, t_{a}\right)\right]^{-1}=\hat{U}_{q}\left(t_{a}, t_{b}\right)
$$

Moreover, on the basis of the definition (20) we can see that the $q$-deformed Hamiltonian $\hat{H}_{q}$ is a $q$-hermitian operator, in the sense that

$$
\hat{H}_{q}^{\dagger} \equiv \hat{H}_{2-q}^{*}=\hat{H}_{q}
$$

The $q$-Schrödinger equation for $\hat{U}_{q}$ can be written as

$$
\begin{aligned}
i \hbar \frac{\partial \hat{U}_{q}\left(t, t_{a}\right)}{\partial t} & =\hat{H}_{q} \hat{U}_{q}\left(t, t_{a}\right) \\
i \hbar \frac{\partial \hat{U}_{q}\left(t, t_{a}\right)^{-1}}{\partial t} & =-\hat{U}_{q}\left(t, t_{a}\right)^{-1} \hat{H}_{q} .
\end{aligned}
$$

Furthermore, the $q$-deformed Schrödinger equation (12) conserves the probability. In fact, if we define the probability density of a single particle in a finite volume as

$$
\rho_{q}=\psi_{q}^{\dagger} \psi_{q} \equiv \psi_{2-q}^{*} \psi_{q}
$$

it is easy to show that

$$
\frac{\partial}{\partial t} \int d^{3} x \rho_{q}=0
$$

Finally, we want to stress that the structure of the Heisemberg's correspondence principle is invariant in the $q$ deformed quantum mechanics. In fact, if we define, as usual, the Heisenberg operator $\hat{O}_{q_{H}}(t)$ as

$$
\hat{O}_{q_{H}}(t)=\hat{U}_{q}\left(t, t_{a}\right)^{-1} \hat{O}_{q}(t) \hat{U}_{q}\left(t, t_{a}\right),
$$

and $\hat{O}_{q}(t)$ is an arbitrary observable in the Schrödinger picture, it is easy to show that

$$
\frac{d \hat{O}_{q_{H}}}{d t}=\frac{i}{\hbar}\left[\hat{H}_{q_{H}}, \hat{O}_{q_{H}}\right]+\frac{\partial \hat{O}_{q_{H}}}{\partial t} .
$$




\section{From non-linear Fokker-Planck equation to non-linear and nonex- tensive Schrödinger equation}

A class of anomalous diffusions are currently described through the non-linear Fokker-Planck equation (NLFPE)

$$
\frac{\partial}{\partial t}[f(t, x)]^{\mu}=-\frac{\partial}{\partial x}\left[J(x)[f(t, x)]^{\mu}-D \frac{\partial}{\partial x}[f(t, x)]^{\nu}\right]_{(30)},
$$

where $D$ and $J$ are the diffusion and drift coefficients, respectively. Tsallis and Bukman [12] have shown that, for linear drift, the time dependence solution of the above equation is a Tsallis-like distribution with $q=1+\mu-\nu$. The norm of the distribution is conserved for all times only if $\mu=1$, therefore we will limit the discussion to the case $\nu=2-q$.

The main characteristic of anomalous diffusion is that the mean squared displacement is not proportional to time $t$ but rather to some power of $t$. If the scaling is faster than $t$, we say that the system is superdiffusive; if it slower than $t$, we say that the system is subdiffusive. Often these processes are strictly connected to memory effects and described by nonlinear evolution equations.

In this section we want to show that it is possible to introduce a different generalization of the Schrödinger equation on the basis of the above NLFPE.

Following, as before, the stochastic quantization and, for simplicity, restricting the discussion to the free case, we obtain the non-linear Schrödinger equation

$$
i \hbar \frac{\partial \psi}{\partial t}=-\frac{\hbar^{2}}{2 m} \frac{\partial^{2}}{\partial x^{2}} \psi^{\nu}
$$

The above equation appears interesting because, differently from Eq.(12), admits a factorized solution $\psi=\phi(t) \varphi(x)$, where $\phi(t)$ obeys to the equation

$$
i \hbar \frac{d \phi(t)}{d t}=E \phi(t)^{\nu}
$$

and the time independent wave function $\varphi(x)$ can be obtained from the $\nu$-deformed eigenvalue equation

$$
\frac{\partial^{2} \varphi(x)^{\nu}}{\partial x^{2}}+k^{2} \varphi(x)=0
$$

where $k=\sqrt{2 m E / \hbar^{2}}$. It is easy to see that the solution of the above equations can be written as

$$
\begin{aligned}
\phi(t) & =\left[1-(1-\nu) i \frac{E}{\hbar} t\right]^{1 /(1-\nu)}, \\
\varphi(x) & =\left[1+\frac{\nu-1}{\sqrt{2 \nu(\nu+1)}} i k x\right]^{1 /(1-\nu)} .
\end{aligned}
$$

Analogously to the previous section, we can introduce the $q$-deformed time evolution operator by means of the following expression

$$
\psi\left(t_{b}\right)=\hat{U}_{q}\left(t_{b}, t_{a}\right) \psi\left(t_{a}\right)
$$

where

$$
\begin{aligned}
\hat{U}_{q}\left(t_{b}, t_{a}\right) & =\exp _{q}\left(-\frac{i}{\hbar} H \frac{t_{b}-t_{a}}{\psi\left(t_{a}\right)^{1-\nu}}\right) \\
& =\left[\exp _{q}\left(-\frac{i}{\hbar} H \frac{t_{a}-t_{b}}{\psi\left(t_{b}\right)^{1-\nu}}\right)\right]^{-1} .
\end{aligned}
$$

It is also interesting to note that by using the property (18) of the $q$-exponential, $\hat{U}_{q}\left(t_{b}, t_{a}\right)$ can be written in a more symmetric form as follows

$$
\hat{U}_{q}\left(t_{b}, t_{a}\right)=\left[\frac{1-(1-\nu) \frac{i}{\hbar} \hat{H} \frac{t_{b}-t_{a}}{\psi\left(t_{a}\right)^{1-\nu}}}{1-(1-\nu) \frac{i}{\hbar} \hat{H} \frac{t_{a}-t_{b}}{\psi\left(t_{b}\right)^{1-\nu}}}\right]^{1 / 2(1-\nu)} .
$$

From the above expressions we can see that the time evolution operator $\hat{U}_{q}\left(t_{b}, t_{a}\right)$ depends explicitly from the initial $a$ and the final state $b$ and not only from the time difference $t_{b}-t_{a}$. Such a behavior is motivated by the non-linear Fokker-Planck equation (30) that implies anomalous diffusion and it is related to memory effects [12]. For this reason the composition law is not satisfied, anomalous diffusion and memory effects imply that $\hat{U}_{q}$ can not be seen as a representation of the abelian group.

Finally, we observe the properties (21) and (22) still hold in the non-linear generalization if we assume, as before, the adjoint operator rule of Eq.(20).

\section{Nonextensive relativistic dynamics}

In the previous sections we have introduced generalized classical and quantum evolution equation in the framework of Tsallis nonextensive conditions. Because of the growing interest to high energy physics applications of nonextensive thermostatistics [6], in this Section we want to study the formulation of the relativistic nonextensive kinetic equations. Let us start defining the basic macroscopic variables in the language of relativistic kinetic theory. Because we are going to describe a non-uniform system in the phase space, we introduce the particle four-flow as $[7,8]$

$$
N^{\mu}(x)=\frac{1}{Z_{q}} \int \frac{d^{3} p}{p^{0}} p^{\mu} f(x, p),
$$

and the energy-momentum flow as

$$
T^{\mu \nu}(x)=\frac{1}{Z_{q}} \int \frac{d^{3} p}{p^{0}} p^{\mu} p^{\nu} f^{q}(x, p),
$$

where we have set $\hbar=c=1, x \equiv x^{\mu}=(t, \mathbf{x}), p \equiv$ $p^{\mu}=\left(p^{0}, \mathbf{p}\right)$ and $p^{0}=\sqrt{\mathbf{p}^{2}+m^{2}}$ is the relativistic energy. The four-vector $N^{\mu}=(n, \mathbf{j})$ contains the probability density $n=n(x)$ (which is normalized to unity) and the probability flow $\mathbf{j}=\mathbf{j}(x)$. The energy-momentum tensor contains the normalized $q$-mean expectation value of the energy density, as well as the energy flow, the momentum and the momentum flow per particle. 
In order to derive a relativistic Boltzmann equation for a dilute system in nonextensive statistical mechanics, we consider the finite volume elements $\Delta^{3} x$ and $\Delta^{3} p$ in the phase space. These volume elements are large enough to contain a very large number of particles but also small enough compared to the macroscopic dimension of the system. In the Lorentz frame, the particle fraction $\Delta N(x, p)$ in the volume $\Delta^{3} x \Delta^{3} p$ can be written as

$$
\Delta N(x, p)=\frac{N}{Z_{q}} \int_{\Delta^{3} x} \int_{\Delta^{3} p} d \Omega f^{q}(x, p),
$$

where $N$ is the total number of particles of the system. With respect to the observer's frame of reference, the above expression becomes

$$
\Delta N(x, p)=\frac{N}{Z_{q}} \int_{\Delta^{3} \sigma} \int_{\Delta^{3} p} d^{3} \sigma_{\mu} \frac{d^{3} p}{p^{0}} p^{\mu} f^{q}(x, p),
$$

where $d^{3} \sigma_{\mu}$ is a time-like three-surface element of a plane space-like surface $\sigma$ [9]. Requiring that the net flow through surface $\Delta^{3} \sigma$ of element $\Delta^{4} x$ vanishes in absence of collisions, we have: $p^{\mu} \partial_{\mu} f^{q}(x, p)=0$. While considering collisions between particles, the Boltzmann equation becomes

$$
p^{\mu} \partial_{\mu} f^{q}(x, p)=C_{q}(x, p) .
$$

$C_{q}(x, p)$ is the $q$-deformed collision term that, under the hypothesis that only binary collisions occur in the gas, can be expressed as

$$
\begin{gathered}
C_{q}(x, p)=\frac{1}{2} \int \frac{d^{3} p_{1}}{p_{1}^{0}} \frac{d^{3} p^{\prime}}{p^{\prime}} \frac{d^{3} p^{\prime}{ }_{1}}{{p^{\prime}}_{1}^{0}}\left\{h_{q}\left[f^{\prime}, f^{\prime}{ }_{1}\right] W\left(p^{\prime}, p^{\prime}{ }_{1} \mid p, p_{1}\right)\right. \\
\left.-h_{q}\left[f, f_{1}\right] W\left(p, p_{1} \mid p^{\prime}, p^{\prime}{ }_{1}\right)\right\}
\end{gathered}
$$

In the above equation, we have set $W\left(p, p_{1} \mid p^{\prime}, p^{\prime}{ }_{1}\right)$ as the transition rate between two particle state with initial fourmomentum $p$ and $p^{\prime}$ and a final state with four-momenta $p_{1}$ and $p_{1}^{\prime} ; h_{q}\left[f, f_{1}\right]$ is the correlation function related to two particles in the same space-time position but with different four-momenta $p$ and $p_{1}$, respectively. The factorization of $h_{q}$ in two single probability distributions (uncorrelated particles at the same spatial point) is the celebrated hypothesis of molecular chaos (Boltzmann's Stosszahlansatz). Thus, the function $h_{q}$ defines implicitly a generalized nonextensive molecular chaos hypothesis.

By assuming the conservation of the energy-momentum in the collisions (i.e. $p^{\mu}+p^{\prime \mu}=p_{1}^{\mu}+p_{1}^{\prime \mu}$ ) and requiring that the correlation function $h_{q}$ is symmetric and always positive ( $\left.h_{q}\left[f, f_{1}\right]=h_{q}\left[f_{1}, f\right], h_{q}\left[f, f_{1}\right]>0\right)$, it is easy to show that collision term satisfies the following property

$$
F[\psi]=\int \frac{d^{3} p}{p^{0}} \psi(x, p) C_{q}(x, p)=0,
$$

if

$$
\psi(x, p)=a(x)+b_{\mu}(x) p^{\mu},
$$

where $a(x)$ and $b(x)$ are arbitrary functions.
By choosing $\psi=$ const. and using the Boltzmann equation (43), then Eq.(45) implies

$$
\frac{\partial}{\partial t} \int d \Omega f^{q}(x, p)=0
$$

and this is nothing else that the conservation of the probability normalization $Z_{q}$. Otherwise, by setting $\psi=b_{\mu} p^{\mu}$, we have from Eq.(45)

$$
\partial_{\nu} T^{\mu \nu}(x)=0
$$

which implies the energy and the momentum conservation.

Let us remark that to have conservation of the probability normalization, energy and momentum, it is crucial that not only the collision term $C_{q}$ be explicitly deformed by means of the function $h_{q}$, but also the streaming term $p^{\mu} \partial_{\mu} f^{q}$. This matter of fact is a direct consequence of the nonextensive statistical prescription of the normalized $q$-mean expectation value and is not taken into account in the non-relativistic formulation of Ref.[10].

The relativistic local $H$-theorem states that the entropy production $\sigma_{q}(x)=\partial_{\mu} S_{q}^{\mu}(x)$ at any space-time point is never negative.

Assuming the validity of Tsallis entropy (1), it appears natural to introduce the nonextensive four-flow entropy $S_{q}^{\mu}(x)$ as follows

$$
S_{q}^{\mu}(x)=-k_{B} \int \frac{d^{3} p}{p^{0}} p^{\mu} f^{q}(x, p)\left[\ln _{q} f(x, p)-1\right] .
$$

On the basis of the above equation the entropy production can be written as

$$
\sigma_{q}(x)=-k_{B} \int \frac{d^{3} p}{p^{0}} \ln _{q} f p^{\mu} \partial_{\mu} f^{q} \equiv-k_{B} F\left[\ln _{q} f\right],
$$

where the second identity follows from the Boltzmann equation (43) and the definition of $F[\psi]$ in Eq.(45). After simple manipulations, Eq.(50) can be rewritten as

$$
\begin{aligned}
& \sigma_{q}(x)=\frac{k_{B}}{8} \int \frac{d^{3} p_{1}}{p_{1}^{0}} \frac{d^{3} p_{1}}{p_{1}^{0}} \frac{d^{3} p^{\prime}}{p^{\prime}} \frac{d^{3} p_{1}{ }^{\prime}}{p^{\prime 0}}\left(\ln _{q} f^{\prime}+\ln _{q} f^{\prime}{ }_{1}-\right. \\
& \left.\ln _{q} f+\ln _{q} f_{1}\right) \times\left\{h_{q}\left[f^{\prime}, f^{\prime}{ }_{1}\right] W\left(p^{\prime}, p^{\prime}{ }_{1} \mid p, p_{1}\right)-\right. \\
& \left.h_{q}\left[f, f_{1}\right] W\left(p, p_{1} \mid p^{\prime}, p^{\prime}{ }_{1}\right)\right\} .
\end{aligned}
$$

By assuming the detailed-balance property, we have that the entropy production is always an increasing function, if $q>0$ and if the function $h_{q}$ satisfies the general condition

$$
h_{q}\left[f, f_{1}\right]=h_{q}\left[\ln _{q} f+\ln _{q} f_{1}\right],
$$

in addition to be symmetric and always positive.

Because the nonextensive formalism reduces to the standard Boltzmann kinetic formulation for $q \rightarrow 1$, it appears natural to postulate the $q$-generalized Boltzmann molecular chaos hypothesis as

$$
h_{q}\left[f, f_{1}\right]=e_{q}\left(\ln _{q} f+\ln _{q} f_{1}\right),
$$


where $e_{q}(x)$ is the Tsallis $q$-exponential function introduced in Eq.(16). Let us note that a similar expression for the function $h_{q}$ was previously introduced in Ref.s [10] and a rigorous justification of the validity of the ansatz (53) can be found only by means of a microscopic analysis of the dynamics of correlations in nonextensive statistics.

\section{Rapidity distribution in relativistic heavy-ion collisions}

In this Section we want to show as nonextensive statistical effects can be very relevant also in the phenomenological interpretation of the high-energy nuclear collisions data. In fact, the quark-gluon plasma close to the critical temperature is a strongly interacting system [6]. For such a system, the colorCoulomb coupling parameter of the QGP can be defined, in analogy to the classical plasma, as

$$
\Gamma \approx C \frac{g^{2}}{r_{0} T}>1
$$

where $C=4 / 3$ or 3 is the Casimir invariant for the quarks or gluons, respectively, $\alpha_{s}=g^{2} /(4 \pi)=0.2 \div 0.5$ and $r_{0}$ is the mean particle distance $r_{0} \simeq n^{1 / 3} \simeq 0.5 \mathrm{fm}$.

Near the phase transition, the interaction range is much larger than Debye screening length (small number of partons in Debey sphere). In fact, $\lambda_{D}=1 / \mu \leq 0.2 \mathrm{fm}$, if we use the non-perturbative estimate $\mu=6 T$. The Coulomb radius for a thermal parton with energy $3 T$ is given by $\left\langle r>=C g^{2} / 3 T=1 \div 6 \mathrm{fm}\right.$. Therefore one obtain $<r>/ \lambda_{D}=5 \div 30$. In other words, memory effects and long-range color interactions give rise to the presence of non-Markovian processes in the kinetic equation affecting the thermalization process toward equilibrium as well as the standard equilibrium distribution.

We are going to study the evolution of the rapidity distribution from a macroscopic point of view by using the nonlinear relativistic Fokker-Planck equation (30) in the space of the rapidity distribution $y$. We show that the observed broad rapidity shape could be a signal of non-equilibrium properties of the system. Let us note that a similar approach, within a linear Fokker-Planck equation, has been previously studied in Ref.[11] by using a linear drift in the space of the rapidity and a free parameter diffusion coefficient. With this choice, the author found a strongly violation of the fluctuation-dissipation theorem. We will see that generalizing the Brownian motion to the relativistic kinetic variables, the standard Einstein relation is satisfied and Tsallis non-extensive statistics emerges in a natural way from the non-linearity of the Fokker-Planck equation.

Basic assumption of our analysis is that the rapidity distribution $y$ is not appreciably influenced by transverse dynamics which is considered in thermal equilibrium. Such hypothesis is well confirmed by the experimental data and adopted in many theoretical works $[13,14,15]$. A crucial rôle in the solution of the above NLFPE plays the choice of the diffusion and the drift coefficients. Such a choice influences the time evolution of the system and its equilibrium distribution.
Since the temperature at freeze-out exceeds $100 \mathrm{MeV}$, quantum statistical effects are negligible and the Boltzmann approximation is usually adopted, therefore the single particle equilibrium distribution is written as

$$
\begin{aligned}
& E \frac{d^{3} N}{d^{3} p} \propto E \exp (-E / T) \\
& \equiv m_{\perp} \cosh (y) \exp \left(-m_{\perp} \cosh (y) / T\right)
\end{aligned}
$$

where $y$ is the rapidity, $m_{\perp}=\sqrt{m^{2}+p_{\perp}^{2}}$ is the transverse mass, $T$ is the temperature. Imposing the validity of the Einstein relation for Brownian particle, we can generalize to the relativistic case the standard expressions of diffusion and drift coefficients as follows [16]

$$
D=\gamma T, \quad J(y)=\gamma m_{\perp} \sinh (y) \equiv \gamma p_{\|},
$$

where $p_{\|}$is the longitudinal momentum and $\tau$ is a common constant. It is easy to see that the above coefficients give us the Boltzmann stationary distribution (5) in the linear Fokker-Planck equation $(q=\nu=1)$. This result cannot be obtained if one assumes a linear drift coefficient as in Ref.[11]. The stationary solution of the NLFPE (30) with $\nu=2-q$ is a Tsallis-like distribution with the relativistic energy $E=m_{\perp} \cosh (y)$. In the light of the above results, we generalize the results of Tsallis-Bukman by searching as a solution of the NLFPE (30) the following time dependent Tsallis-like distribution

$$
\begin{aligned}
& f_{q}\left(y, m_{\perp}, t\right)= \\
& \left\{1-(1-q) \beta(t) m_{\perp} \cosh \left[y-y_{m}(t)\right]\right\}^{1 /(1-q)}
\end{aligned}
$$

The unknown functions $\beta(t)=1 / T(t)$ and $y_{m}(t)$ have been derived by means of numerical integration of Eq.(30) with initial $\delta$-function condition depending on the value of the experimental projectile rapidities. The rapidity distribution at fixed time is then obtained by numerical integration over the transverse mass $m_{\perp}$ (or transverse momentum) as follows

$$
\frac{d N}{d y}(y, t)=c \int_{m}^{\infty} m_{\perp}^{2} \cosh (y) f_{q}\left(y, m_{\perp}, t\right) d m_{\perp},
$$

where $c$ is the normalization constant fixed by the total number of the particles. Rapidity spectra calculated from (58) will ultimately depend on two parameters: the "interaction" time $\tau=\gamma t$ and the non-extensive parameter $q$. Therefore, no more free parameters are used in this analysis respect to previous theoretical studies $[14,15]$. 


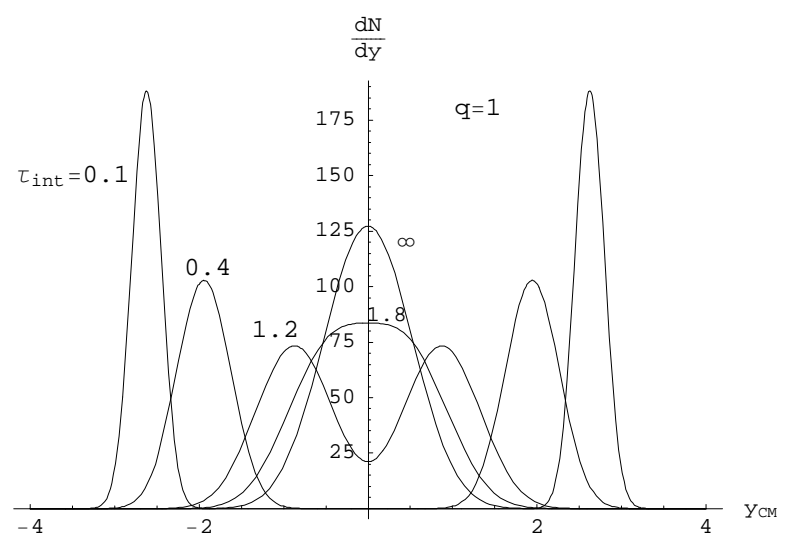

Figure 1. Shape of the rapidity distribution at different integration time $\tau_{i n t}$ for the undeformed case $(q=1)$

The obtained spectra are normalized to 164 protons and the beam rapidity is fixed to $y_{\mathrm{cm}}=2.9$ (in the c.m. frame) [13]. In Fig. 1, we show the shape of the rapiditity distribution at different integration time $\tau_{\text {int }}$ of the linear FokkerPlanck equation $(q=1)$. In Fig. 2, we report the time evolution of the rapidity distribution corresponding to the integration of the NLFPE with $q=1.25$ (the value of the $q$ parameter used to fit the data).

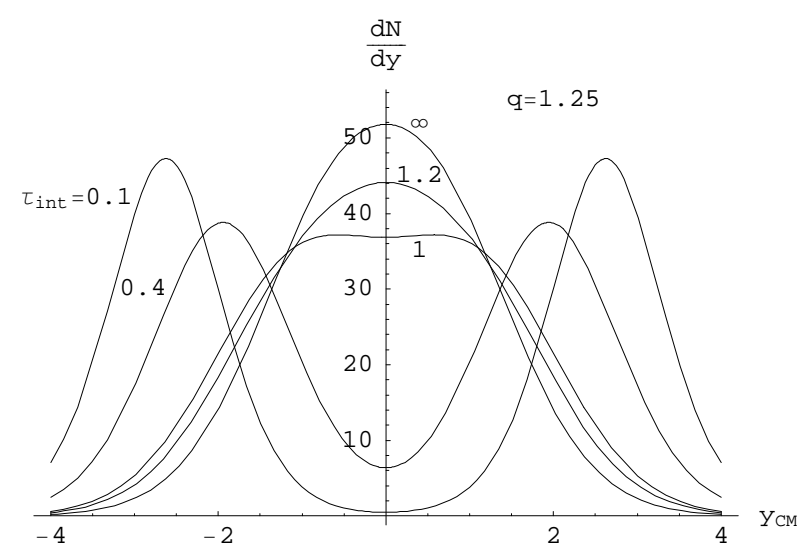

Figure 2. The same of Fig. 1 for $q=1.25$

In Fig. 3, we show the calculated rapidity spectra compared with the experimental data. The full line corresponds to the NLFPE solution (58) at $\tau_{\text {int }}=0.82$ and $q=1.25$; the dashed line corresponds to the solution of the linear case $(q=1)$ at $\tau_{i n t}=1.2$. Imposing the validity of the fluctuation-dissipation theorem, it is not possible to reproduce the experimental rapidity shape at any time. Only in the non-linear case $(q \neq 1)$ exists a (finite) time for which the obtained rapidity spectra well reproduces the broad experimental shape. A value of $q \neq 1$ implies anomalous superdiffussion in the rapidity space, i.e., $\left[y(t)-y_{M}(t)\right]^{2}$ scale like $t^{\alpha}$ with $\alpha>1$ [12].

A complete description of the applicability of nonextensive statistical effects to high-energy heavy ion collisions lies out the scope of this paper. However, we want to outline that an analysis of the transverse pion momentum spectra and the net proton rapitity distribution measured at RHIC is under investigation.

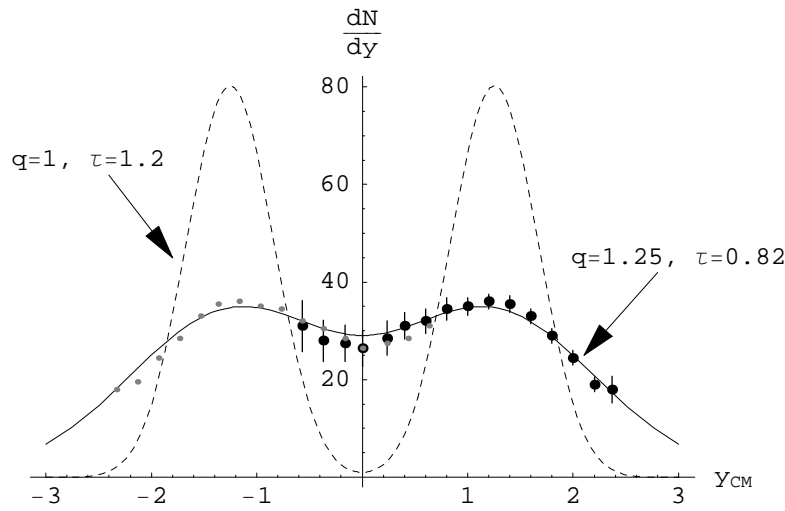

Figure 3. Rapidity spectra for net proton production $(p-\bar{p})$ in central $\mathrm{Pb}+\mathrm{Pb}$ collisions at $158 \mathrm{~A} \mathrm{GeV} / \mathrm{c}$ (grey circles are data reflected about $y_{c m}=0$ ) [13]. Full line corresponds to our results by using a non-linear evolution equation $(q=1.25)$, dashed line corresponds to the linear case $(q=1)$

\section{Conclusion}

There is an increasing evidence that the generalized nonextensive thermodynamics can be considered as the more appropriate basis of a theoretical framework to deal with several physical phenomena where long-range interactions, longrange memory effects and/or fractal space-time constraints are present. A considerable variety of physical applications involve microscopic quantum and/or relativistic effects. The main effort of this paper is to study an appropriate generalization of the quantum dynamics and relativistic kinetic equation in the framework of the Tsallis nonextensive thermostatistics. We have introduced two kinds of generalized Schrödinger equations which satisfy the basic assumptions of the quantum mechanics under appropriate operator properties which depend on the deformation parameter $q$. Furthermore, we have studied a nonextensive generalization of the relativistic Boltzmann equation and of the nonlinear Fokker-Planck equation. The above evolution equations have been applied to the study of the nonequilibrium rapidity distribution in relativistic heavy-ion collisions obtaining a very good agreement with the experimental results.

\section{References}

[1] C. Tsallis, J. Stat. Phys. 52, 479 (1988). See also http://tsallis.cat.cbpf.br/biblio.htm for a regularly updated bibliography on the subject.

[2] C. Tsallis, R.S. Mendes, and A.R. Plastino, Physica A 261, 534 (1998)

[3] S. Martínez, F. Nicolás, F. Pennini, and A. Plastino, Physica A 286, 489 (2000). 
[4] S. Martínez, F. Pennini, and A. Plastino, Phys. Lett. A 278, 47 (2000).

[5] L. Borland, Phys. Lett. A 245, 67 (1998).

[6] W.M. Alberico, A. Lavagno, and P. Quarati, Eur. Phys. J. C 12, 499 (2000); Nucl. Phys. A 680, 94c (2001).

[7] A. Lavagno, Phys. Lett. A 301, 13 (2002).

[8] A. Drago, A. Lavagno, and P. Quarati, Physica A 344, 472 (2004).

[9] S.R. Groot, W.A. van Leeuwen, and Ch. G. van Weert, Relativistic Kinetic Theory (North-Holland, Amsterdam, 1980).

[10] J.A.S. Lima, R. Silva, and A.R. Plastino, Phys. Rev. Lett. 86, 2938 (2001)
[11] G. Wolschin, Eur. Phys. J. A 5, 85 (1999); Phys. Rev. C 69, 024906 (2004).

[12] C. Tsallis, D.J. Bukman, Phys. Rev. E 54, R2197 (1996).

[13] H. Appelshäuser et al. (NA49 Collaboration), Phys. Rev. Lett. 82, 2471 (1999).

[14] I.G. Bearden et al. (NA44 Collaboration), Phys. Rev. Lett. 78, 2080 (1997).

[15] P. Braun-Munzinger et al., Phys. Lett. B 344, 43 (1995); B 356, 1 (1996).

[16] A. Lavagno, Physica A 305, 238 (2002). 